\title{
A Method of MPPT Control Based on Power Variable Step-size in Photovoltaic Converter System
}

\author{
Xu Hui-xiang ${ }^{1}$, Li Nian-qiang ${ }^{2}$ \\ ${ }^{1}$ School of Information Science and Engineering,University of Jinan, jinan 250022,China \\ ${ }^{2}$ School of Information Science and Engineering,University of Jinan, jinan 250022, China
}

\begin{abstract}
Since the disadvantage of traditional MPPT algorithms of variable step-size, proposed power tracking based on variable step-size with the advantage method of the constant-voltage and the perturb-observe $(\mathrm{P} \& \mathrm{O})^{[1-3]}$. The control strategy modify the problem of voltage fluctuation caused by perturb-observe method, at the same time, introducing the advantage of constant-voltage method and simplify the circuit topology. With the theoretical derivation, control the output power of photovoltaic modules to change the duty cycle of main switch. Achieve the maximum power stabilization output, reduce the volatility of energy loss effectively, and improve the inversion efficiency ${ }^{[3,4]}$. Given the result of experimental test based theoretical derivation and the curve of MPPT when the prototype work.
\end{abstract}

Key Words: MPPT, variable step-size, constant-voltage, P\&O, inversion efficiency, circuit topology

\section{Introduction}

The maximum power point tracking(MPPT) is the important element of photovoltaic converter system. Its rationale is that control the duty cycle of main power switch by the acquisition value of voltage and current to achieve the maximum power point (MPP), and stability output. The control algorithm can be divided roughly into three methods: constant-voltage methods, perturb-observe methods, and incremental conductance ${ }^{[5]}$. The reference[4] proposed a method of variable step-size incremental conductance based on Boost topology, used $|d p / d u|$ as the coefficient of variable step-size. But this method need collect current value, increase the complicated structure of circuit topology. The reference[6] proposed a prediction-based variable step perturbation and observation method to avoid misjudge. But this method still need to disturbance voltage.

In this paper, through the analysis of traditional
MPPT algorithms of variable step-size and the advantage and disadvantage methods of the constant-voltage and perturb-observe, proposed a method of power variable step-size combining constant-voltage method and perturb-observe method, on the premise of no voltage disturbance, without input current collect input current to control the output power from photovoltaic modules with power variable step-size. Just collect the voltage value, simplify the circuit topology.

\section{The analysis of advantage and disadvantage of three methods}

The output curve of photovoltaic panels with the same temperature different light intensity and the same light intensity different temperature has shown in figure- $1^{[5]}$. We can found that the MPP distribute near the dotted line in the picture. 

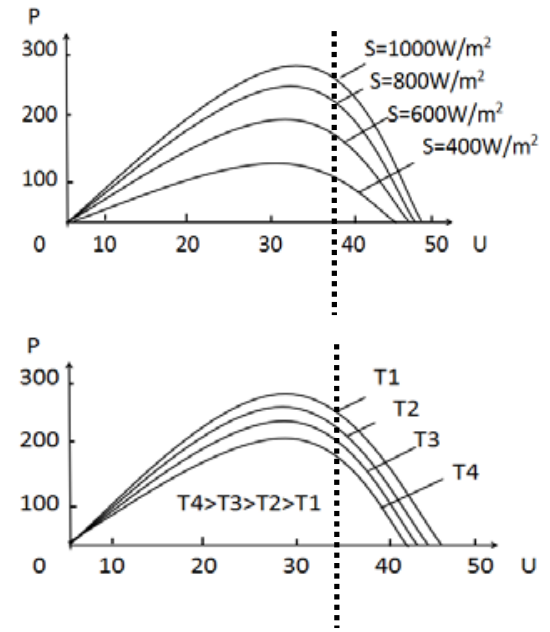

Fig.1 The curve of the same temperature different light intensity and the same light intensity different temperature

The thought control of constant-voltage method is fixed the input voltage on the dotted line to achieve the MPP, with low control precision, low efficiency, and can not tracking in real time ${ }^{[2,7]}$.

Compared to constant-voltage method, the method of perturb-observe is more widely used. Judgment the change of power before and after and disturbance voltage to achieve $\mathrm{MPPT}^{[5,8]}$. But the disturbance is a kind of interference will occur voltage fluctuation, and reduce the converter efficiency.

The traditional variable step-size incremental conductance achieve MPPT through two ways: P-U derivation and power variation rate . The former must collect current value for the accurate calculation will complicate the circuit topology. The latter need to judgment and determine multiple stages, its will increase the complicate of algorithm with a complexity circuit topology.

\section{A Method Of Mppt Control Based On Power Variable Step-Size}

\subsection{Control principle}

Aim at the issue of complicate circuit based on $|d p / d u|$ as variable step-size, and the problem of the constant-voltage and the perturb-observe, proposed a method of MPPT control based on power variable step-size, without current collect, and no voltage disturbance, computing power increment $(\Delta p)$ by the value of voltage, control output power of photovoltaic to achieve MPPT. The figure-2 shown the curve of P-V. According to the method of constant-voltage, set the range of MPP, which is usually about 0.8 times of open-circuit voltage. Instead of $|d p / d u|$. used $\Delta p$ as the parameter of variable step-size, closer it gets to the MPP, smaller the step-size. When the $\Delta p$ is zero, collect voltage and judgment whether within the 0.8 times rage of open-circuit voltage to avoid misjudgment. Change the duty cycle of the main switch by control output power of photovoltaic, accordingly change the input voltage, and adjust the output load. When get the MPP, because of the power constantly, the input voltage will not change, reducing the voltage fluctuation.

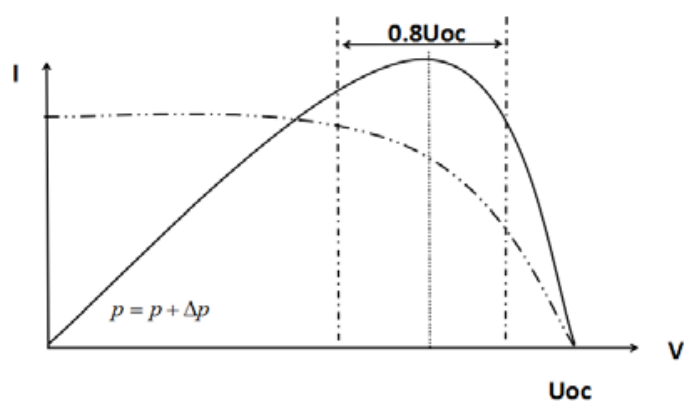

Fig.2 The curve of P-V

\subsection{Theoretical Derivation}

The figure- 3 shown the equivalent output circuit of the photovoltaic ${ }^{[9]}$. The Cin is a large capacity electrolyte capacitor use for balance the instantaneous power of DC side and Ac side. According to the law of conservation of energy, the output energy of photovoltaic $(E p v)$ is equal to the sum of the input energy of converter $(E i)$ and the energy $(E c)$ stored in $\mathrm{Cin}$ :

$$
E c=E p v-E i
$$

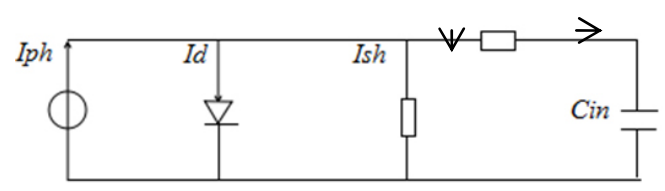

Fig.3 equivalent output circuit of the photovoltaic 
Let $T p$ as a power cycle, $P p v$ is the average output power of photovoltaic, $P_{i}$ is the input power of converter, in a power cycle, the above formula can be written as:

$\frac{1}{2} C_{i n} U_{t+T p}^{2}-\frac{1}{2} C_{i n} U_{t}^{2}=P_{p v} T p-\int_{t}^{t+T p} p_{i} d t$

(2)

In the formula, $U_{t}$ is the capacitor voltage in $\mathrm{t}$ moment, $U_{t+t p}$ is the capacitor voltage after a power cycle.

Set $t_{0}$ to $t_{1}, t_{1}$ to $t_{2}$, the average output power was $P_{1}, P_{2}$, and the electrolytic capacitor voltage at the time of $t_{0}, t_{1}$ and $t_{2}$ are respectively $U_{0}, U_{1}$ and $U_{2}$. Take $t_{1}=t_{0}+T_{p}, t_{2}=t_{1}+T_{p}$ into the formula 2 :

$$
\begin{aligned}
& \frac{1}{2} C_{\text {in }} U_{1}^{2}-\frac{1}{2} C_{\text {in }} U_{0}^{2}=P_{1} T_{p}-\int_{t_{0}}^{t_{1}} p_{i} d t \\
& \frac{1}{2} C_{\text {in }} U_{2}^{2}-\frac{1}{2} C_{\text {in }} U_{1}^{2}=P_{2} T_{p}-\int_{t_{1}}^{t_{2}} p_{i} d t
\end{aligned}
$$

Because the grid energy is equal in two consecutive power cycles, so

$$
\int_{t_{0}}^{t_{1}} p_{i} d t=\int_{t_{1}}^{t_{2}} p_{i} d t
$$

subtract (3) from (4):

$$
\Delta p=\Delta P_{p v}=P_{2}-P_{1}=\frac{C i n}{2 T p}\left(U_{2}^{2}-2 U_{1}^{2}+U_{0}^{2}\right)
$$

$\frac{C i n}{2 T p}$ is a constant, $\Delta p$ is power variation, take $\Delta p=U_{2}^{2}-2 U_{1}^{2}+U_{0}^{2}$ as the parameter of variable step-size. The calculation formula of duty cycle D and switch cycle $T s$ is[10]:

$$
\begin{gathered}
D=\frac{U g}{N \bullet U p v+U g} \\
T s=L p \bullet P o p\left(\frac{\sin \varphi_{G}}{U p v \bullet D}\right)^{2}
\end{gathered}
$$

In formula (8), the Pop is the output peak power of converter and the $U p v$ is the input voltage of converter, control the $T s$ and $U p v$ by change the value of Pop, adjusting the duty cycle of switch and matching output impedance. The MPPT control flow chart of the method shown in figure-4.

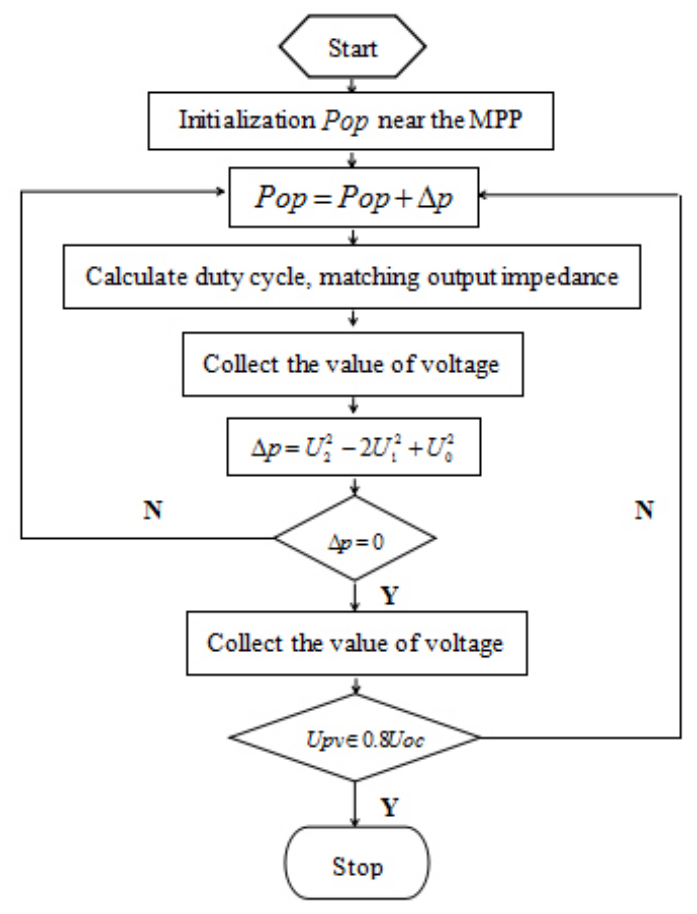

Fig.4 The MPPT control flow chart

\section{Prototype testing and experimental curves}

In this paper, the converter rated capacity is 230VA, input DC $30 \mathrm{~V}$, output $\mathrm{AC} 220 \mathrm{~V}$, the main power MOSFET used IRF4321, and the parasitic capacitance Coss $=390 p F$, the primary induction of the main power switches is $5.4 \mu \mathrm{H}$, the leak inductance is $0.2 \eta H$, the ratio of turns is $1: 6$, the clamp capacitance $\mathrm{Ca}=3.3 n F$, the leak inductance recovery resistance $\mathrm{R} 1=100 \mathrm{~K}$, the clamp diode used ES1D, the switch tube in secondary side used $17 N 80$, and the commutation diode used C2D05120.

The experiment waveform are shown in figure-5. Figure-5(a) shown the Simulation of light intensity and temperature change curve in a day[9,11-12]. The figure-5(b) shown the Prototype maximum power point tracking in real time and the efficiency of MPPT. We can clearly see that, the power point stable near the MPP and the efficiency achieve 99.3\%. The figure-6(c) shown the grid voltage waveform with the peak value is about $330 \mathrm{~V}$, and very smooth. The output converter efficiency can reach $96.2 \%$. 


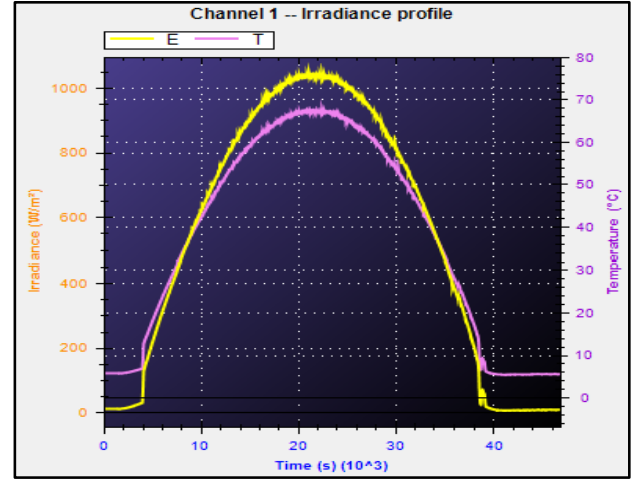

(a) The curve of light intensity and temperature

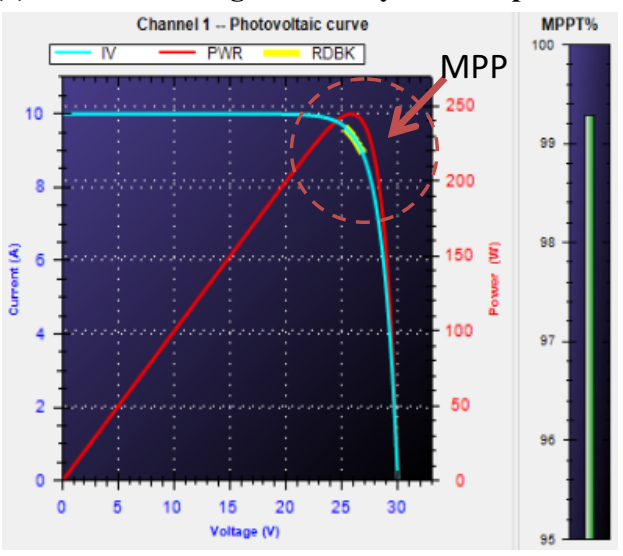

(b) The curve of MPPT and the efficiency

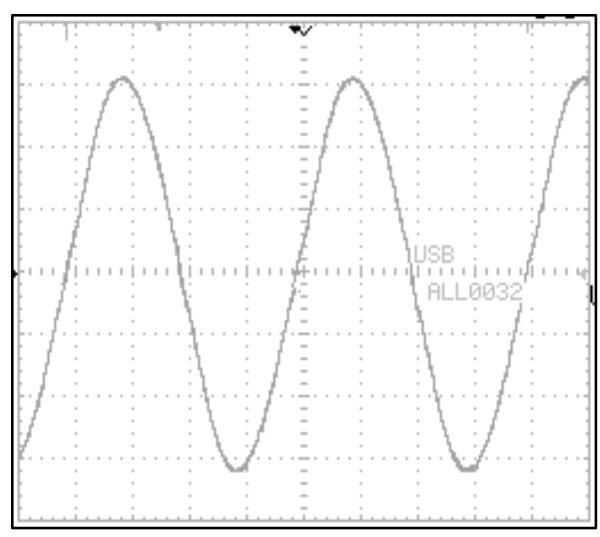

$(\mathrm{t}=10 \mathrm{~ms} /$ lattice, peak value $=100 \mathrm{~V} /$ lattice $)$

(c) The converter AC output voltage

Fig. 5 Prototype testing and experimental curves

\section{Conclusion}

The Method of MPPT Control Based on Power Variable Step-size combining the advantage of constant-voltage and the perturb-observe, remove the circuit topology of current acquisition, simplify the circuit topology, reduce the algorithm requirements to the bottom of hardware. Improved the issue of voltage fluctuation caused by the method of perturb-observe, and the complicate of circuit topology and algorithm in traditional variable step-size incremental conductance.

\section{References}

1. WANG Ya-nan, YANG Xu-hong, WANG Jun-cheng, etc. One Novel Variable Step-size Strategy of MPPT for Photovoltaic System[J]ELECTRIC DRIVE,2015(01):54-57

2. XIONG Yuansheng, YU Li1, XU Jianming. MPPT control of photovoltaic generation system combining constant-voltage method with perturb-observe method[J]. Electric Power Automation Equipment,2009(06):85-88

3. Xue Yang, Wang Sha. Fuzzy Control Based on P\&O Applied In Photovoltaic Maximum Power Tracking[J]. ACTA ENERGIAE SOLARIS SINICA,2014(09):1622-1626

4. LAI Dong-sheng, YANG Ping. Variable Step-size Incremental Conductance Used in Maximum Power Point Tracking Control of PV Power System[J]. Power Electronics,2012(3):40-42

5. XU Peng-wei, LIU Fei, LIU Bang-yin, DUAN Shan-xu. Analysis, Comparison and Improvement of Several MPPT Methods for PV System[J]. Power Electronics,2007(05):3-5

6. LIU Zeng-huan, LI Jie. Application of power prediction-based variable step perturbation and observation method in PV MPPT[J]. Manufacturing Automation,2015(02):2-4

7. YU Yun-jun, ZHANG Xiang, WANG Shi-sheng, ZHANG Yan-fei. Review of incremental conductance method for photovoltaic power generation MPPT $[\mathrm{J}]$. Chinese Journal of Power Sources, 2014(12):2440-2444

8. Wu Junjuan, Jiang Yida, Wang Qiang, Sun Xiaofeng. An Improved MPPT Control Algorithm of PV System[J]. ACTA ENERGIAE SOLARIS SINICA,2012(03):478-482

9. Yang Yongheng Zhou Keliang. Photovoltaic Cell Modeling and MPPT Control Strategies[J]. Transactions of China Electrotechnical Society, 2011(12):40-45 
10. GU Junyin1, WU Hongfei2, CHEN Guocheng1, XING Yan 2. Soft-switching Grid-connected PV Inverter With Interleaved Flyback Topology[J]. Proceedings of the CSEE, 2011(12):40-45

11. Wu Dazhong, Wang Xiaowei. A Photovoltaic MPPT Fuzzy Controlling Algorithm[J]. ACTA ENERGIAE SOLARIS SINICA,2011(06):808-813

12. SUN Hang, DU Haijiang, JI Yingxu, YANG Bo. Photovoltaic distributed MPPT mechanism analysis and simulation study[J]. Power System Protection and Control,2015(01):48-54 